\title{
Synthesis of $\mathrm{TiO}_{2}$ nanoparticles by hydrolysis and peptization of titanium isopropoxide solution
}

\author{
S. Mahshid ${ }^{1}$, M. Sasani Ghamsari ${ }^{2}$, M. Askari ${ }^{1}$, N. Afshar $^{3}$, S. Lahuti ${ }^{3}$ \\ ${ }^{1}$ Department of Material Science \& Eng., Sharif Industrial University, 11365-9466, Tehran, Iran \\ ${ }^{2}$ Solid State Laser Div., Laser Research Center, AEOI, 11365-8486, Tehran, Iran \\ ${ }^{3}$ Material \& Ionic radiation staff, AEOI, Karage, Iran \\ E-mail: msasani@aeoi.org.ir
}

\begin{abstract}
Titanium dioxide nanoparticles have been prepared by hydrolysis of titanium isopropoxide. An aqueous solution with varied $\mathrm{pH}$ and peptizing the resultant suspension was used to prepare $\mathrm{TiO}_{2}$ nanopowder with a narrow size distribution. The synthesized powder was examined by X-ray diffraction, scanning electron microscope and transmission electron microscope. The as-prepared powder entirely consisted of anatase crystalline phase with a particle size of $7.6 \mathrm{~nm}$. Only powder obtained from the solution of $\mathrm{pH}=2$ had a fine particle size with spherical morphology. The anatase to rutile transformation occurred at the temperatures lower than $600{ }^{\circ} \mathrm{C}$.
\end{abstract}

Keywords: hydrolysis, peptization, titanium isopropoxide, crystallinity, $\mathrm{TiO}_{2}$.

Manuscript received 07.01.06; accepted for publication 29.03.06.

\section{Introduction}

One of the most recent nanomaterials that has been attracted a great attention due to its unique properties is titanium dioxide. $\mathrm{TiO}_{2}$ powders possess interesting optical, dielectrical, and catalytical properties, which results in industrial applications such as pigments, fillers, catalyst supports, and photocatalysts [1-6].

Control of the size, shape, and structure of the colloidal precursor is an important factor in determining the properties of the final material. Titania powders have been obtained either directly from titanium-bearing minerals or by precipitation from solutions of titanium salts or alcoxides. The most common procedures were based on the hydrolysis of acidic solutions of Ti (1V) salts [7]. In addition, gas-phase oxidation reactions of $\mathrm{TiCl}_{4}$ and hydrolysis reactions of titanium alcoxides were used to generate finely separated, high purity $\mathrm{TiO}_{2}$ powders. However, these powders have generally lacked the properties of uniform size, equated shape, and unagglomerated state desired for quantitative studies of colloidal phenomena [7]. Recently, several techniques were reported for synthesizing titania nanoparticles through controlled nucleation and growth processes in dilute Ti (IV) solutions. Among them are the sol-gel, hydrothermal and peptization methods, with advantage of low reaction temperature [8-13]. It has been demonstrated that the initial nanostructure of titania precursors prepared by hydrolyzing titanium alcoxide strongly affects the crystallization behavior and characteristics of the final powder [14]. The peptization process has the same advantage of flexibility as the solgel method, in which the reaction can be carried out at a molecular level by heating the solution or using peptization agent $[14,15]$. In this research work, we have tried to optimize preparation conditions for providing the narrow size distribution of the nanometer $\mathrm{TiO}_{2}$ powders.

\section{Experimental}

In this work, the precursor solution was a mixture of $5 \mathrm{cc}$ titanium isopropoxide, TTIP (97\%, supplied by Aldrich Chemical) and 15cc Isopropanol (99\%, supplied by Merck). A $250 \mathrm{cc}$ solution of distilled water with varied $\mathrm{pH}$ was used as the hydrolysis catalyst. The desired $\mathrm{pH}$ value of the solution was adjusted by adding $\mathrm{HNO}_{3}$ or $\mathrm{NH}_{4} \mathrm{OH}$. The gel preparation process started when both solutions were mixed together under vigorous stirring. Hydrolysis of TTIP offered a turbid solution which heated up to $60-70{ }^{\circ} \mathrm{C}$ for almost $18-20$ hours. The resultant suspension was white-blue and opaque with high viscosity. The prepared precipitates were washed with ethanol and dried for several hours at $100{ }^{\circ} \mathrm{C}$ and finally annealed at a temperature ranging from 200 to $800^{\circ} \mathrm{C}$ for 2 hours.

Several techniques were used to characterize the powders. Powder X-ray diffraction (Philips PW 1800) 
was used for crystal phase identification and estimation of the average crystallite size. The particle size and morphology of the powder were observed on Philips XL 30 scanning electron microscope (SEM) and Philips 200 transmission electron microscope (TEM).

\section{Results and discussion}

The preparation of $\mathrm{TiO}_{2}$ colloids in the nanometer range can be effectively carried out through the hydrolysis and condensation of titanium alcoxides in aqueous media. In the presence of water, alcoxides hydrolyze and subsequently polymerize to form a three-dimensional oxide network. These reactions can be schematically represented as follows:

$\mathrm{Ti}(\mathrm{OR})_{4}+4 \mathrm{H}_{2} \mathrm{O} \rightarrow 2 \mathrm{Ti}(\mathrm{OH})_{4}+4 \mathrm{ROH}$ (hydrolysis),

$\mathrm{Ti}(\mathrm{OH})_{4} \rightarrow \mathrm{TiO}_{2} x \mathrm{H}_{2} \mathrm{O}+(2-x) \mathrm{H}_{2} \mathrm{O}$ (condensation),

where $\mathrm{R}$ is ethyl, $i$-propyl, $n$-butyl, etc.[16]. Even so, hydrolysis in the presence of excess water is rapid and completes within seconds. To moderate the high reactivity, alcoxides are usually diluted in alcohol prior to mixing with water. The size, stability, and morphology of the sol produced from alcoxides is strongly affected by the water-to-titanium molar ratio $\left(r=\left[\mathrm{H}_{2} \mathrm{O}\right] /[\mathrm{Ti}]\right)$. One may distinguish two regimes: (i) at low $r$ values $(r \leq 10)$, one obtains spherical, relatively monodisperse particles with diameters of 0.5 to $1 \mathrm{~mm}$; (ii) at higher $r$ values, the particles formed are colloidal unstable and precipitate in the form of large aggregates. These aggregates can be chemically peptized to final sizes that are usually less than $100 \mathrm{~nm}$ in diameter [1719]. The formation of colloidal $\mathrm{TiO}_{2}$ at high $r$ ratios is of great interest, because of the small size of particles that are formed under these conditions. In addition, the $\mathrm{pH}$ of solution has a strong influence on the size distribution of nanoparticles. When the $\mathrm{pH}$ level of the solution is higher than 2, a white suspension of rough precipitants is formed immediately as the hydrolysis reactions is occurred. Otherwise, when the $\mathrm{pH}$ level of the solution is 2, a homogenious suspension of fine particles is formed. After heating at $60-70{ }^{\circ} \mathrm{C}$ for almost $18-20 \mathrm{hr}$, the volume of the suspension decreases to $50 \mathrm{cc}$ and a whiteblue gel suspension is produced. After being washed with ethanol and dried at $100{ }^{\circ} \mathrm{C}$ in a vacuum system for $2 \mathrm{~h}$, a yellow-white powder is obtained. In Fig. 1, illustrated are the XRD patterns of $\mathrm{TiO}_{2}$ powders prepared from an acidic solution $(\mathrm{pH}=2)$ and calcined at various temperatures under atmospheric condition for $2 \mathrm{~h}$. All the samples are crystalline.

The XRD pattern of the powder dried at $100{ }^{\circ} \mathrm{C}$ (Fig. 1a) is characteristic of anatase $\mathrm{TiO}_{2}$. It shows that peptization can accelerate the crystallization and shift it to the temperatures lower than even $100{ }^{\circ} \mathrm{C}$. The temperature observed in our result is much lower than that achieved by others [20]. The XRD peaks become sharper as the calcination temperature is increased.

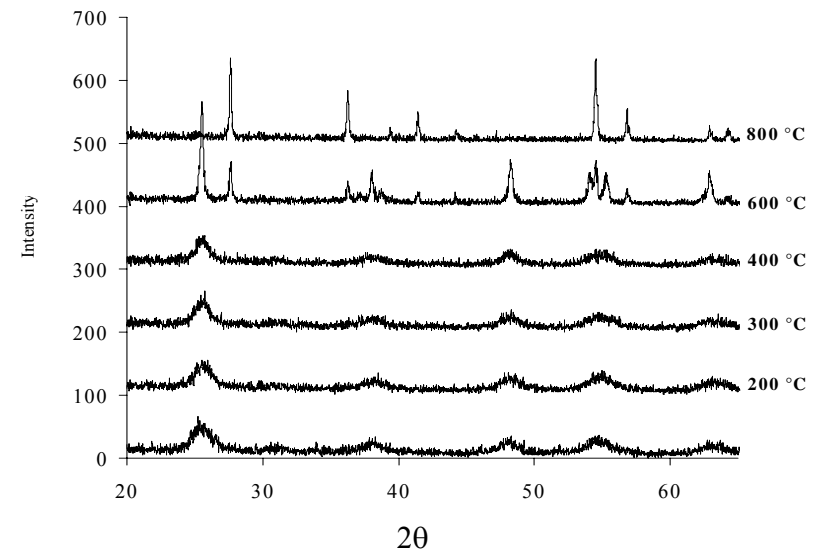

Fig. 1. XRD patterns of powders prepared at different calcination temperatures ( $\mathrm{A}$ - anatase, $\mathrm{R}$ - rutile).

When the calcination temperature is $600{ }^{\circ} \mathrm{C}$, rutile phase quite sharp peaks are observed. This suggests that there is a phase transition from anatase to rutile at the temperatures lower than $600^{\circ} \mathrm{C}$, which agrees with previous experiments [21]. The residual anatase phase still exists in the samples up to $800^{\circ} \mathrm{C}$. At $800^{\circ} \mathrm{C}$, the transition to the rutile phase is complete. Crystallinity of the calcined titania powder at various temperatures were studied. The crystallite size was estimated from the Debye-Scherrer equation [22-25] using the XRD line broadening as follows:

$B=\frac{k \lambda}{s \cos \theta}$,

where $s$ is the crystallite size, $\lambda$ is the wavelength of the $\mathrm{X}$-ray radiation $(\mathrm{CuK \alpha}=0.15406 \mathrm{~nm}), k$ is a constant taken as $0.94, \theta$ is the diffraction angle and $B$ is the line width at half maximum height. The (101) plane diffraction peak is used for anatase and (110) peak for rutile. The effect of calcination temperatures on the crystallinity of $\mathrm{TiO}_{2}$ was reported in Table. As seen in Table, the smallest crystallites are obtained from the hydrolysis of TTIP in the acidic solution of $\mathrm{pH}=2$ and after drying the powder at $100^{\circ} \mathrm{C}$. At higher calcination temperatures, the formed crystallites are larger in size, which can be attributed to the thermally promoted crystallite growth. The size of the anatase crystallites increases from 7.6 to $38.7 \mathrm{~nm}$ when the calcination temperature is raised to $600^{\circ} \mathrm{C}$. In this case, the rutile phase shows quite different behavior in which the formed rutile phase is somewhat larger than that of anatase. Furthermore, the crystallite size is $47.5 \mathrm{~nm}$ at $600{ }^{\circ} \mathrm{C}$, while it increases only slightly to $53.4 \mathrm{~nm}$ at $800^{\circ} \mathrm{C}$. These results suggest that the rutile nuclei are more tending to growth than anatase ones and the growth process starts right after nucleation of rutile phase. Fig. 2 shows the SEM micrographs of $\mathrm{TiO}_{2}$ particles prepared at different calcination temperatures. As shown in Fig. 2a, the as-prepared powder consists of spherical particles with poor agglomeration, and the aggregation takes place during the particle growth process at higher temperatures (Fig. 2b). 

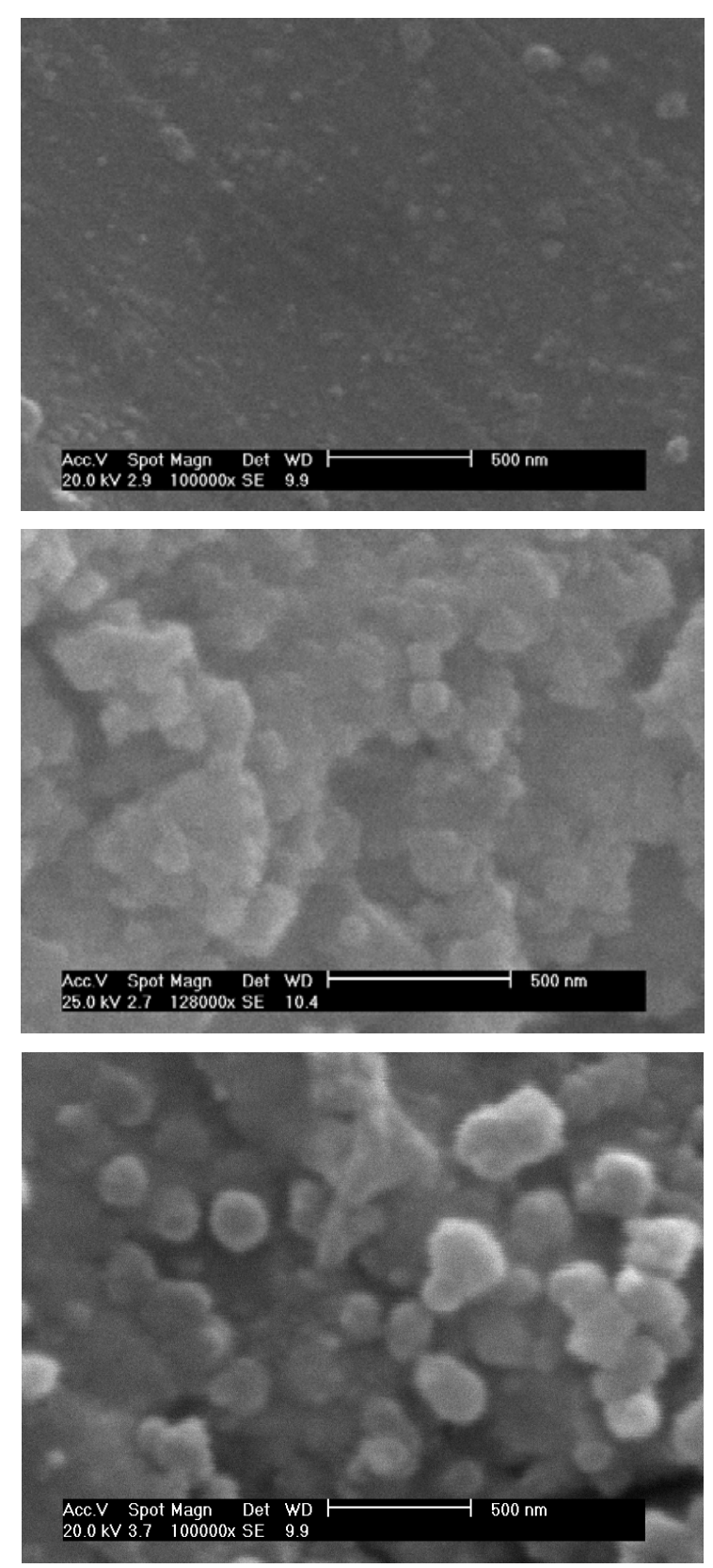

Fig. 2. SEM micrographs of powders prepared at different temperatures: (a) as-prepared, (b) calcined at $200{ }^{\circ} \mathrm{C}$, (c) $400{ }^{\circ} \mathrm{C}$.

Table. XRD results for $\mathrm{TiO}_{2}$ particles calcined at various temperatures for $2 \mathrm{~h}$.

\begin{tabular}{|c|c|c|}
\hline \multicolumn{2}{|c|}{ Crystallite size $(\mathrm{nm})$} & $\begin{array}{c}\text { Calcined temp. } \\
\left({ }^{\circ} \mathrm{C}\right)\end{array}$ \\
\hline Rutile & Anatase & Dried gel \\
\hline- & 7.6 & 200 \\
\hline- & 8.9 & 300 \\
\hline- & 14.2 & 400 \\
\hline 47.5 & 28.4 & 600 \\
\hline 53.4 & 38.7 & 800 \\
\hline
\end{tabular}

It is observed that, at the higher calcination temperatures, the larger particle size with spherical morphology is obtained. For the sample calcined at $400{ }^{\circ} \mathrm{C}$ (Fig. 2c), the particle size is almost $100 \mathrm{~nm}$. Grain boundaries are clearly observed in the SEM micrographs of the samples calcined at the high temperatures. From the SEM photographs of samples prepared under different $\mathrm{pH}$ values, it was revealed that the spherical morphology was a specification of the prepared powder under acidic solution of $\mathrm{pH}=2$ and it was not seen in other samples. The TEM image and the select-area electron diffraction (SAED) pattern of the asprepared sample are shown in Figs $3 a$ and b, respectively. It is obviously shown that the as-prepared powder is completely crystalline and entirely consists of anatase phase. Because of the poor contrast in the micrograph, it is difficult to exactly measure the size of the primary spherical particles accurately. From the micrograph, their diameter is estimated to be below $10 \mathrm{~nm}$, which is in good agreement with the XRD results. In Fig 3b, shown is the SARD pattern of asprepared $\mathrm{TiO}_{2}$ particles that disappear at $100{ }^{\circ} \mathrm{C}$. The first four rings are assigned to the (101), (004), (200), (005) reflections of the anatase phase. The SAED studies are in good agreement with the XRD measurements.
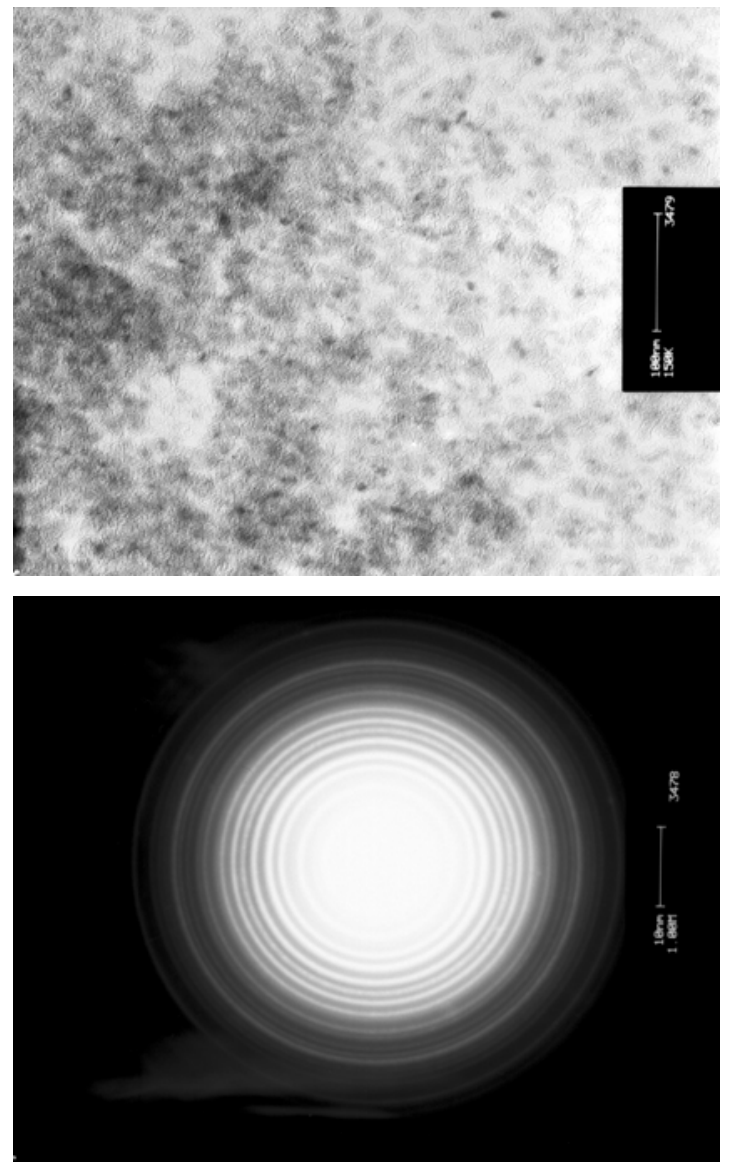

Fig. 3. TEM (a) and SAED (b) photographs of as-prepared sample dried at $100{ }^{\circ} \mathrm{C}$ for 3 hours. 


\section{Conclusions}

A nanocrystalline $\mathrm{TiO}_{2}$ powder can be prepared by the hydrolysis of titanium-isopropoxide alcoholic solution and then peptization of the resultant suspension up to $60 \ldots 70{ }^{\circ} \mathrm{C}$ for $18 \ldots 20$ hours. According to the particle size obtained from the Debye-Scherrer equation, the powder obtained from a solution of $\mathrm{pH}=2$ consists of very fine anatase crystallites even at a temperature lower than $100^{\circ} \mathrm{C}$. When the powder is treated thermally at $400^{\circ} \mathrm{C}$, the diameter of nanoparticles is approximately $28 \mathrm{~nm}$ and still consists of pure anatase phase. As the calcination temperature is increased, the particle size increases. Rutile phase is formed at calcination temperatures below $600{ }^{\circ} \mathrm{C}$ and grows slightly when heated up to $800^{\circ} \mathrm{C}$. Powder morphology in these criteria is almost spherical, which is due to acidic condition that prevents agglomeration.

\section{References}

1. C.J. Barbe, F. Arendse, M. Grätzel // J. Amer. Ceram. Soc. 80, p. 3157 (1997).

2. M. Ahmed, Y.A. Attia // J. Non-cryst. Solids $\mathbf{1 8 6}$ p. 402 (1995).

3. M. Ferroni, V. Guidi, G. Martinelli, P. Nelli // Nanostructured Mater. 7, p. 709 (1996).

4. J.E. Rekoske, M.A. Barteau // J. Phys. Chem. B 101, p. 1113 (1997).

5. Y.C. Yeh, T.Y. Tseng, D.A. Chang // J. Amer. Ceram. Soc. 73, p. 1992 (1990).

6. A. K. Atta, P. K. Biswas, and D. Ganguli // Mater. Lett. 15, p. 99-103 (1992).

7. E.A. Barringer and H.K. Bowen // Longmuir 1, p. 414-420 (1985).
8. J.A. Ayllon, A. Figueras, S. Garelik, L. Spirkova, J. Durand, L. Cot // J. Mater. Sci. Lett. 18, p. 1319 (1999).

9. Y.F. Chen, C.Y. Lee, M.Y. Yen, H.T. Chiu // J. Crystal Growth 247, p. 363-370 (2003).

10. J. Yang, S. Mei, J.M.F. Ferreira // Mater. Sci. \& Eng. C 15, p. 183-185 (2001).

11. E.J. Kim, S.H. Hahn // Mater. Lett. 49, p. 244-249 (2001).

12. X.Z. Ding, X.H. Liu // J. Alloys \& Compounds 248, p. 143-145 (1997).

13. S.R. Dhage, V.D. Choube, V. Samuel, V. Ravi // Mater. Lett. 58, p. 2310-2313 (2004).

14. T. Zeng, Y. Qiu, L. Chen // Materials Chemistry and Physics 56, p. 163-170 (1998).

15. B.L. Bischoff, M.A. Anderson // Chem. Mater. 7, p. 1772 (1995).

16. D. Vorkapic and T. Matsoukas // J. Amer. Ceram. Soc. 81, p. 2815-2820 (1998).

17. J.-L. Look and C.F. Zukoski // Ibid. 75, p. 1587-95 (1992).

18. J.-L. Look and C.F. Zukoski // Ibid. 78, p. 21-32 (1995).

19. V.J. Nagpal, R.M. Davis, and J.S. Riffle // Colloids Surf. 87, p. 25-31 (1994).

20. E.J. Kim, S.-H. Hahn // Mater. Lett. 49, p. 244 (2001).

21. K.P. Kumar, K. Keizer, A.J. Burggraaf // J. Mater. Chem. 3, p. 1141 (1993).

22. B. Li, X. Wang, M. Yan, L. Li // Materials Chemistry and Physics 78, p. 184-188 (2002).

23. L. Ying, L.S. Hon, T. White, R. Withers and L.B. Hai // Materials Transactions 44, p.1328-1332 (2003).

24. M.A. Barakat, G. Hayes, S. Ismat Shah // J. Nanoscience and Nanotechnology 5, p. 1-7 (2005).

25. G. Oskam, A. Nellore, R. Lee Penn, P.C. Searson // J. Phys. Chem. B 107, p. 1734-1738 (2003). 